\title{
Thoracic chordoma with epidural extension: a case report of total en bloc spondylectomy
}

\section{—N. Kawahara, K. Tomita, H. Murakami, S. Demura}

Department of Orthopaedic Surgery, Kanazawa University, Kanazawa, Japan

This presentation was made during the 13th Argospine Symposium, in 2009

No funds were received in support of this study

61-year-old male
5 months history of
back pain
Paraparesis
(Frankel Grade D)
No history of carcinoma
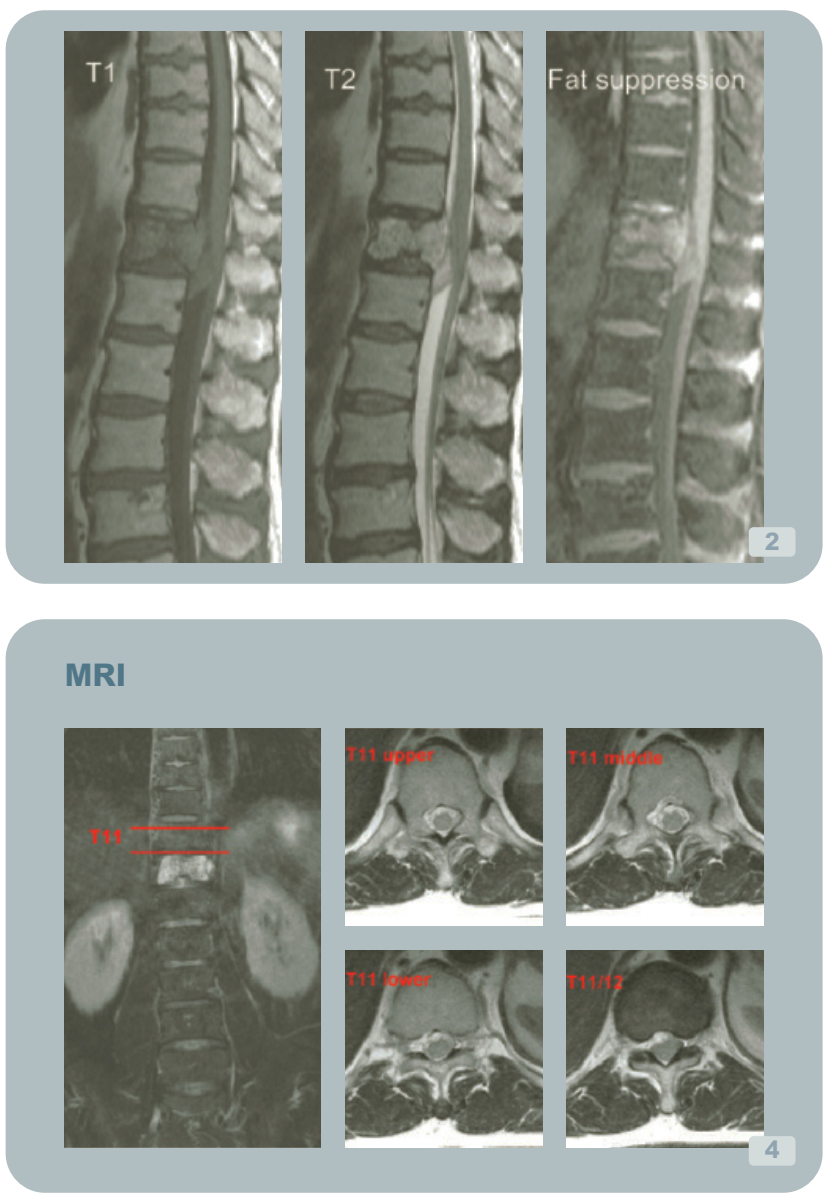

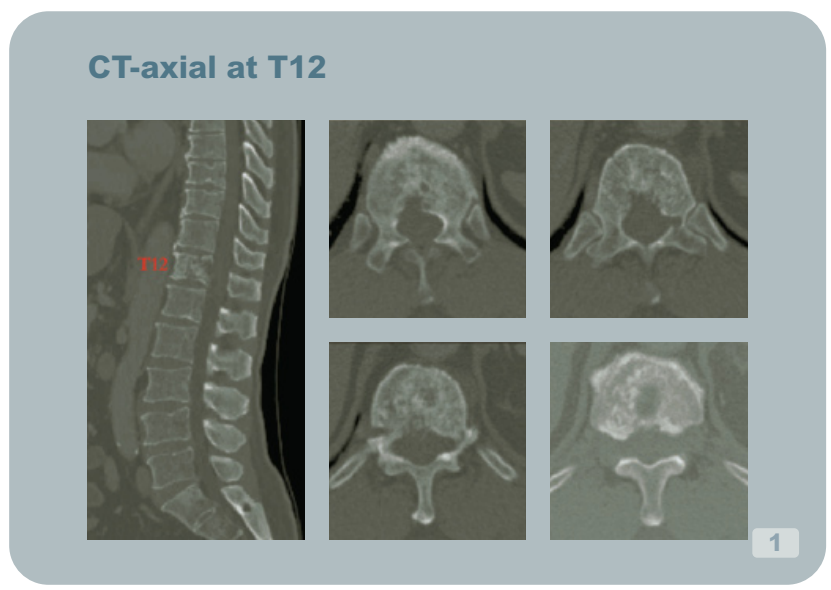

T1 fat suppression enhancement (+)

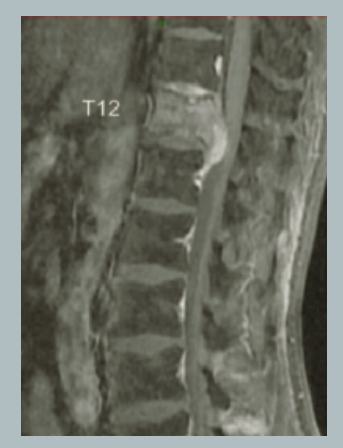

3

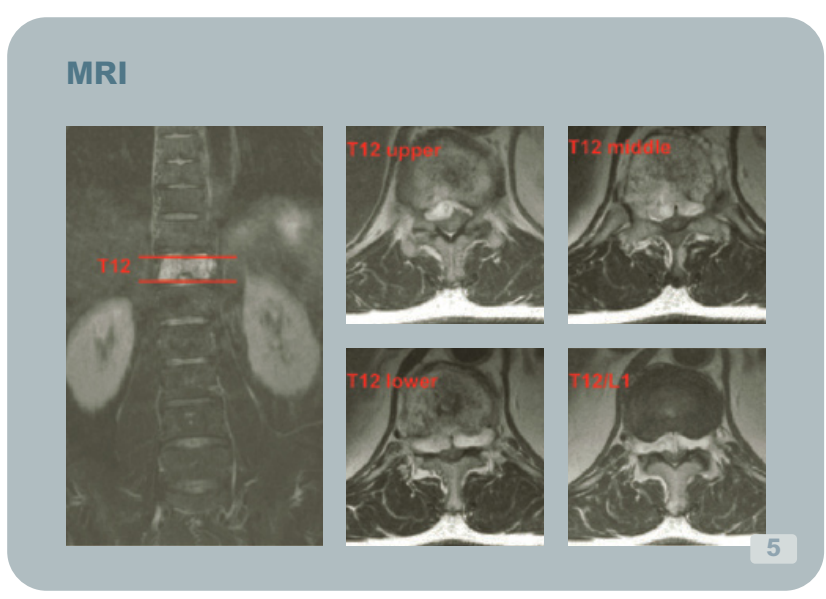


MRI
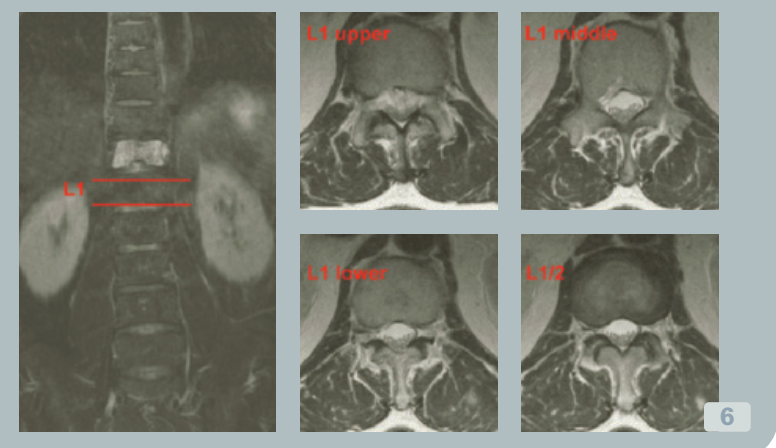

What is the diagnosis?

Interactive vote

1. Solitary metastasis: origin unknown

$19 \%$

2. Malignant fibrous histiocytoma

$13 \%$

3. Chondrosarcoma

$8 \%$

4. Chordoma

5. Plasmacytoma

$24 \%$

6. Lymphoma

$12 \%$

What is the treatment?

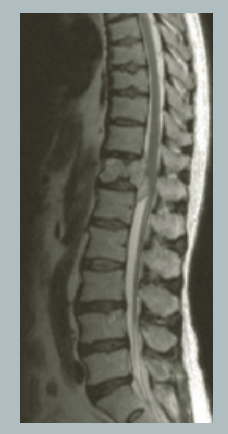

Case: 61-year-old male

Diagnosis: Chordoma at T12

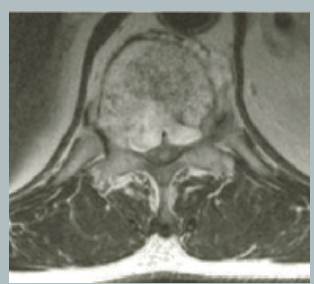

10

\section{Chordoma of the mobile spine}

"The only treatment protocol associated with patients that are continuously disease-free (CDF) at follow-up longer than 5 years is margin free en bloc resection." [1]

(1) Boriani S et al. (2006) Chordoma of the mobile spine: fifty years of experience. Spine 31(4): 493-503
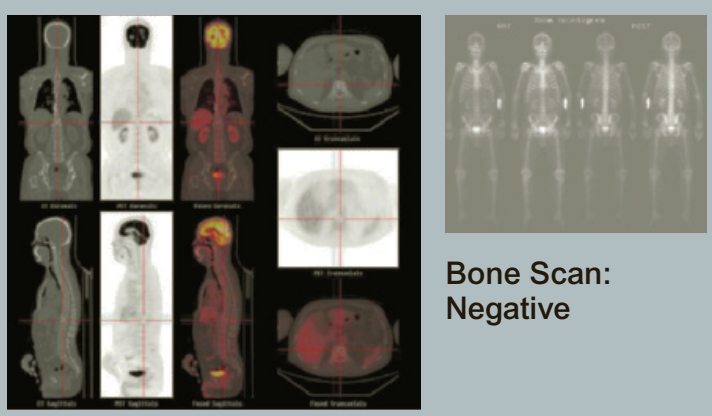

Bone Scan:

Negative

PET: Negative

Needle biopsy:

Chordoma

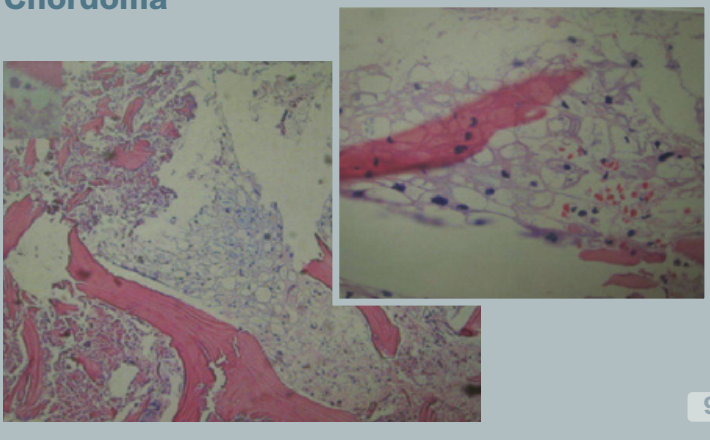

What is the treatment?

Interactive vote

1. Palliative surgery

= $3 \%$

2. Piecemeal excision including margin $=5 \%$

3. En bloc resection with tumor margin

$92 \%$

How to remove the tumor vertebra?
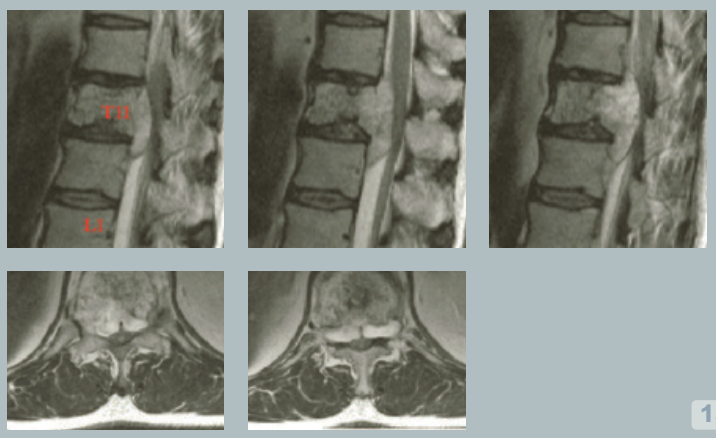
How to remove

the tumor vertebra?

1. Posterior laminectomy followed by anterior corpectomy

2. Anterior dissection followed by posterior removal $42 \%$

3. Posterior approach alone $17 \%$

Which level of the anterior column should be osteotomized?

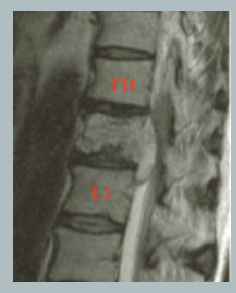

Left

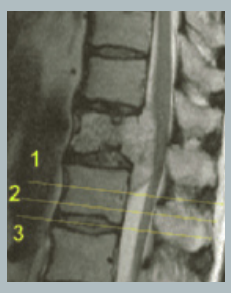

Middle

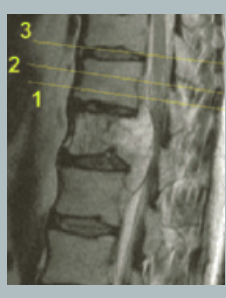

Right

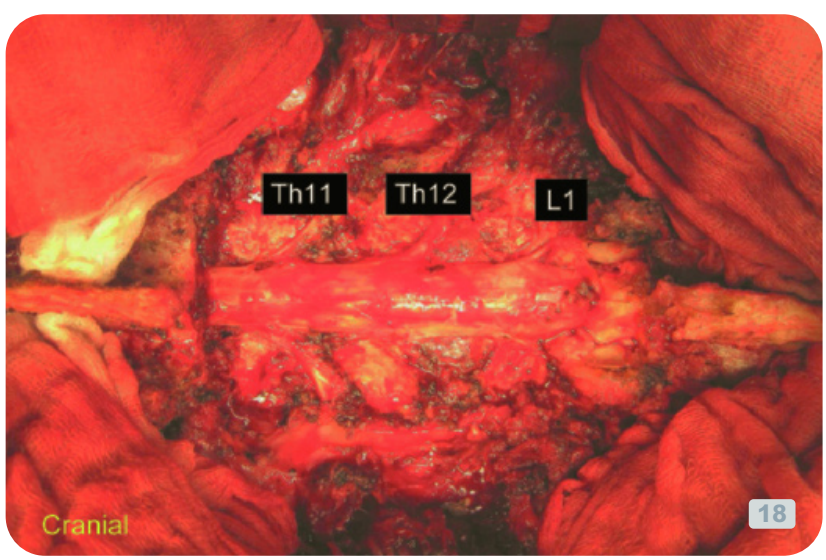

Which level of the anterior Interactive vote column should be osteotomized?

Which level of the anterior column should be osteotomized?

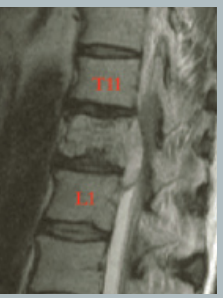

Left

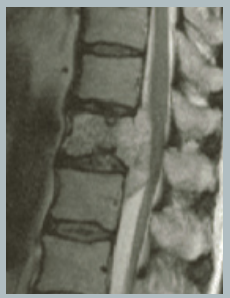

Middle

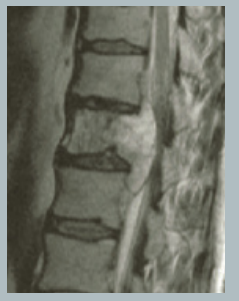

Right
15

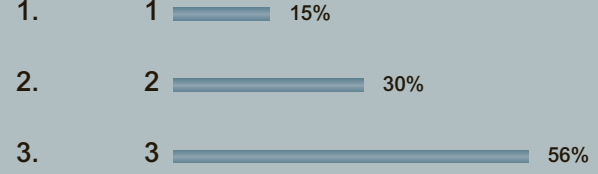

How to remove the tumor vertebra keeping margin without injuring the spinal cord?
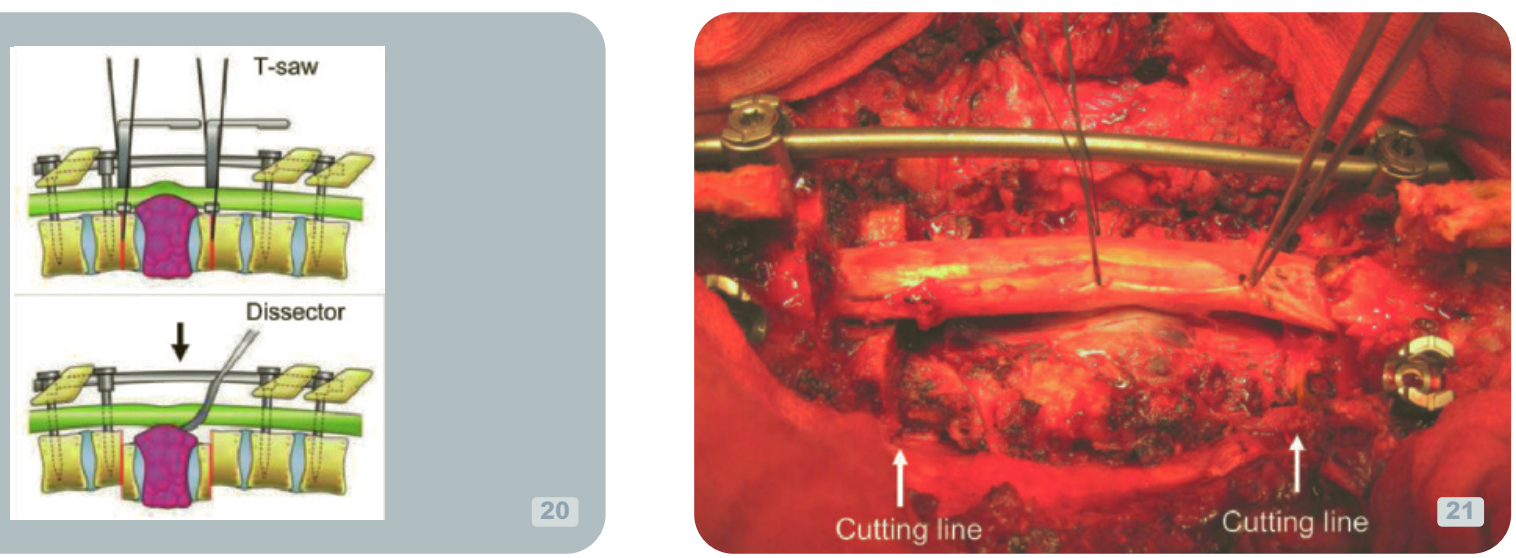

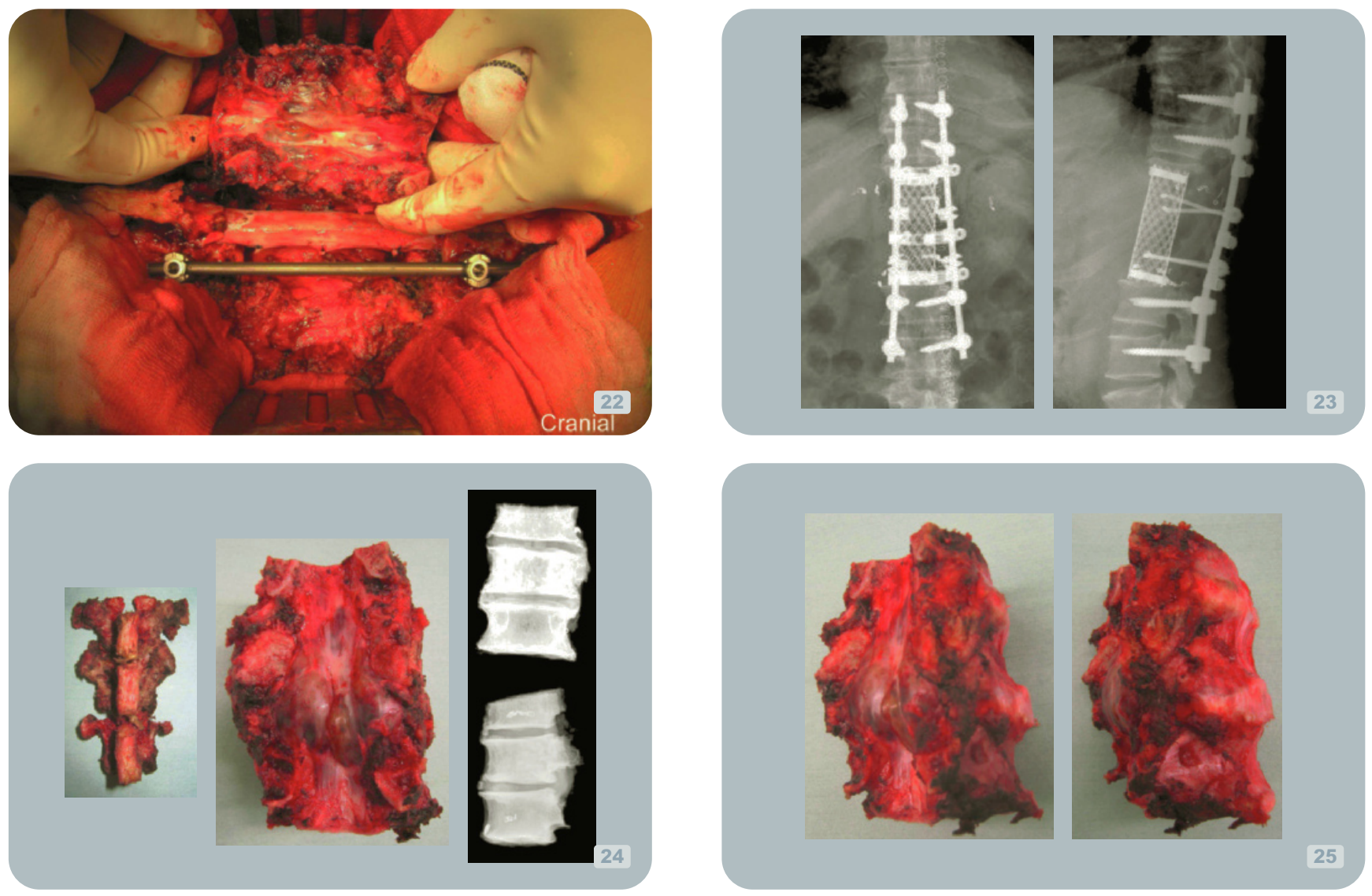

\section{Our message}

This procedure allowed:

- en bloc marginal resection

with no neurologic degradation

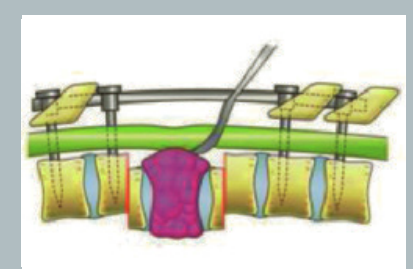

in our patient with encapsulated epidural tumor. 